\title{
Avaliação e Mensuração de Resultados em Gestão de Pessoas: uma análise da produção científica brasileira em revistas
}

\section{Evaluation and Measurement of Results in People Management: an analysis of Brazilian scientific production in journals}

\author{
JHONY PEREIRA MORAES \\ ANGELA BEATRIZ BUSATO SCHEFFER \\ SIDIMAR MEIRA SAGAZ \\ Universidade Federal do Rio Grande do Sul
}

\section{RESUMO}

O presente artigo teve como objetivo identificar o perfil dos artigos científicos no tema Avaliação e Mensuração de Resultados em Gestão de Pessoas publicados em revistas brasileiras. Salienta-se a relevância desta pesquisa, que procurou retratar o cenário brasileiro de produção científica nesta área especifica de Gestão de Pessoas, não tendo sido encontrado estudo semelhante envolvendo as últimas décadas. Para a realização deste artigo, foi executada uma pesquisa descritiva do tipo bibliométrica. A abordagem foi quantitativa e qualitativa e envolveu 34 artigos. Os resultados permitiram observar que a maioria dos artigos publicados foram produzidos em instituições da região Sudeste, principalmente na USP, são de orientação qualitativa, tiveram crescimento a partir de 2006. Observou-se, também, momentos em termos de pesquisas: (1) a busca por evidenciar a realidade das organizações, as transformações ocorridas e as esperadas da área, e apresentar de que forma pode-se estar direcionando uma atuação mais estratégica; (2) a discussão sobre o alinhamento das práticas de Gestão de Pessoas e a mensuração dos resultados e (3) a proposição de modelos que possam clarificar as relações entre desempenho e as práticas de Gestão de Pessoas, com temas como 
capacidades organizacionais e recursos sendo trazidos. Campo com potencial ainda de estudos.

Palavras-Chave: Gestão de Pessoas, Avaliação e Mensuração de Resultados, Bibliometria.

\section{Abstract}

The aim of this article was to identify the profile of the scientific articles in the topic Evaluation and Measurement of Results in People Management published in Brazilian journals. We highlight the relevance of this research, which sought to portray the Brazilian scenario of scientific production in this specific area of People Management, no similar study involving the last decades. For the accomplishment of this article, a descriptive research of the bibliometric type was executed. The approach was quantitative and qualitative and involved 34 articles. The results showed that most of the published articles were produced in institutions of the Southeast region, mainly in the USP, are of qualitative orientation, had growth from 2006. It was also observed moments in terms of researches: (1) the seeks to demonstrate the reality of organizations, the changes that have occurred and those expected in the area, and present how a more strategic action can be directed; (2) discussing the alignment of People Management practices and measuring results; and (3) proposing models that can clarify the relationship between performance and People Management practices, with themes such as organizational capacities and resources being brought. Field with potential of studies.

Keywords: People Management, Evaluation and Results Measurement, Bibliometrics.

\section{INTRODUÇÃo}

As mudanças ocorridas no mercado ao longo dos anos impulsionam as organizações a repensarem o papel e a estrutura da área de Gestão de Pessoas (GP), buscando sua maior articulação estratégica com os objetivos organizacionais. Nesse sentido, tornou-se importante olhar para as políticas e práticas de gestão de pessoas que estão sendo construídas, identificando as possibilidades de alinhamento da área com a estratégia empresarial e os resultados que estão sendo alcançados (CASTRO; SILVA, 2012; TINTI; COSTA; VIEIRA; 
CAPPELLOZZA, 2017). Diante disso, o estudo acerca da avaliação e mensuração de resultados em Gestão de Pessoas é oportuno. Pensar sobre a questão dos resultados em GP é, assim, considerar sobre como tem sido visto e operacionalizado esse alinhamento entre os objetivos organizacionais, as pessoas e as políticas e práticas de GP em seus contextos específicos. Mais ainda, é avaliar os impactos efetivos desse alinhamento no desempenho das organizações.

Ao longo dos anos, autores (CHAHAL; JYOTI; RANI, 2016; PEISENIECE; VOLKOVA, 2010; PIETSCH, 2007; STEEN; WELCH; MCCORMACK, 2011; ULRICH, 1997; WARECH; TRACEY, 2004; ZEHIR et al., 2016), em nível internacional, e em âmbito nacional (MASCARENHAS; MANZINI, 2008; MURITIBA et al., 2010; OLIVEIRA; ALBUQUERQUE; MURITIBA, 2003), buscaram ampliar o escopo de estudo sobre avaliação e mensuração de resultados em GP. Apresentaram análises de cenários sobre o tema, seus possíveis desdobramentos e impactos no desempenho organizacional, assim como na vida dos colaboradores. Entretanto, maior parte da produção científica sobre o tema concentra-se em discussões de âmbito internacional. No Brasil, os trabalhos que revisaram a produção científica no país sobre o tema foram desenvolvidos na primeira década dos anos 2000 e utilizaram limitadores de tempo e de revistas científicas ou anais de eventos para a inclusão dos trabalhos analisados.

Considerando tais pressupostos, este artigo tem por objetivo analisar o perfil e a evolução da produção científica brasileira sobre avaliação e mensuração de resultados em GP publicadas em revistas nacionais. Assim, de modo específico, procurou-se identificar a cronologia das publicações, os periódicos, principais instituições e autores, natureza dos estudos e cenários dos estudos; levantar os principais mecanismos/indicadores utilizados nos estudos; e demonstrar as contribuições/possíveis avanços e entraves ao processo. Espera-se com este estudo oferecer um panorama atual acerca do tema abordado.

O presente artigo estrutura-se da seguinte forma: parte do entendimento das mudanças da área de GP em direção a uma atuação mais estratégica e passa a discutir como vem sendo tratado o tema da avaliação e mensuração dos resultados de GP. Após, apresenta 
o método utilizado e discute os resultados, para então finalizar com as considerações gerais.

\section{REFERENCIAL TEÓRICO}

\subsection{Gestão Estratégica de Pessoas}

A crescente competividade dos mercados e suas consequentes transformações suscitaram a importância da atuação da área de GP na estratégia de negócio, exigindo a elaboração de práticas e políticas de GP que pudessem dar melhor resposta aos desafios organizacionais. A GP, dentro de um contexto estratégico, passou a ser considerada um diferencial para as empresas (ASHTON; HAFFENDEN; LAMBERT, 2004; BOXALL; PURCELL, 2003; ULRICH, 1998), pressupondo, nesse fazer estratégico da área, uma ação mais contextual, integrada a processos, capaz de atender aos resultados, focada nas pessoas e na estratégia de negócios como forma de fomentar a competitividade organizacional (LEGGE, 2005; LENGNICK-HALL et al., 2009). Nesse sentido, há uma busca da área por agregar valor à organização.

No Brasil, autores como Albuquerque (1999; 2002), Almeida, Teixeira e Martinelli (1993), Fischer (2002) e Lacombe e Bendassolli (2004), entre outros, procuraram trazer essa discussão sobre a evolução da área em nosso país, enquanto outros deram ênfase as dificuldades, aos avanços ou mesmo ao que pouco mudou na área, destacando-se Cançado et al. (2017), Coda, César e Garcia (2002) e Tanure, Evans e Cançado (2010). Segundo os autores, tal processo no Brasil ocorreu de forma mais marcante a partir da década de 1990.

Distintamente da gestão tradicional de pessoas, a GEP baseia-se em traços organizacionais que contemplem a organização do trabalho e sua realização, os valores e a filosofia organizacional e os sistemas de recursos humanos, estando esses orientados para o estreitamento do potencial dos indivíduos à estratégia da empresa (PAROLIN; ALBUQUERQUE, 2011). Para a obtenção de efetivos resultados para a empresa, a GP deve integrar os planos estratégicos da organização (ALMEIDA; TEIXEIRA; MARTINELLI, 1993; SILVA; LEITE; RODRIGUES, 2016), por sua vez, destacam ações que visem a organização e manutenção de equipes orientadas pelos objetivos 
organizacionais. (LACOMBE; CHU, 2008) ressaltam que na GEP as pessoas são consideradas recursos e direcionadas à vantagem competitiva. Além disso, ressalta o alinhamento interno (entre políticas e práticas) e sua integração com o negócio (alinhamento externo).

Como características, Parolin e Albuquerque (2009) afirmam que na GEP processos como recrutamento e seleção objetivam potencializar o desenvolvimento dos candidatos, tanto interno quanto externamente. Pressupõe também o consentimento de profissionais sobre o desempenho de diferentes papeis, facilidade de comunicação e espírito colaborativo. Os treinamentos assumem a função de desenvolverem competências somativas ao desempenho organizacional, agregando valor ao trabalho, assim como atender as suas necessidades em decorrência da introdução de práticas e processos inovadores e das mudanças que poderão vir a ocorrer. Citam-se outras características, como: descentralização do processo decisório, a sinergia, criação de cenários alternativos, o desenvolvimento e motivação humanos (ALMEIDA; TEIXEIRA; MARTINELLI, 1993); análise do ambiente de negócio da empresa (BIANCHI; QUISHIDA; FORONI, 2017); e abordagem de temas como inovação, governança corporativa, mudanças no mercado de trabalho, internacionalização de empresas e avaliação de resultados de GP (LEITE; ALBUQUERQUE, 2013).

Entre o discurso e a entrega efetiva do esperado para a área, torna-se, então, imprescindível avaliar e mensurar a contribuição da área, o que será então discutido.

\subsection{Avaliação e Mensuração de Resultados em Gestão de Pessoas}

As mudanças nas últimas décadas fizeram com que a GP enfrentasse pressões para assegurar sua legitimação econômica (PIETSCH, 2007). Com a propagação do conceito de GEP, a necessidade de resultados econômicos a partir das práticas de GP se tornou mais forte (MASCARENHAS; MANZINI, 2008). Com isso, surge a necessidade da GP acompanhar, mensurar e avaliar o impacto de suas práticas no desempenho das organizações (MASCARENHAS; MANZINI, 2008).

De modo geral, a relação entre as práticas de GP e os resultados do negócio é construída sobre a premissa de que a melhor implanta- 
ção e utilização de práticas de GP deve se correlacionar com maiores resultados do negócio. Becker, Huselid e Ulrich (2001) trazem que as complementaridades entre as dimensões de ajuste interno e externo são fundamentais para o desempenho organizacional. Entretanto, tal relação vem sendo um dos grandes desafios para os profissionais da área que operam segundo a orientação de uma GEP, uma vez que parte desses profissionais perpetuam o mito de que os resultados em GP são essencialmente intangíveis e de difícil mensuração. Mascarenhas e Manzini (2008) e Oliveira e Oliveira (2011) trazem o estudo de Combs et al. (2006) que apontou para a complexidade dessa relação, ao considerar que "a função de gerenciar pessoas permeia toda a organização e, por conseguinte, afeta de maneira complexa e simultânea todos os seus resultados, sejam recursos humanos, organizacionais, financeiros ou de mercado" (p. 655).

A avaliação dos resultados de GP possibilita distinguir práticas de gerenciamento de pessoal que auxiliam na criação de valor econômico daquelas que simplesmente são neutras e/ou possibilitam perdas no valor econômico das organizações. Warech e Tracey (2004) recomendam o uso de sistemas de alto desempenho em GP. Evidências sugerem que investimentos nessas práticas de trabalho possibilitam menor rotatividade de funcionários e maior produtividade e desempenho financeiro corporativo (HUSELID, 1995).

$\mathrm{Na}$ tentativa por mensurar e avaliar os resultados das ações de GP, organizações tem buscado suas métricas. Assis (2001) aborda indicadores, que podem ser tanto quantitativos ou qualitativos, quanto demográficos (quantidade e/ou disponibilidade de recursos humanos por meio de métricas). Podem ser financeiros quando medem o investimento que as empresas fazem nas pessoas; operacionais/indicadores de processo, de desempenho ou de eficiência (consumo de recursos). Dentre os indicadores quantitativos mais usados na área estão os índices de absenteísmo e rotatividade, bem como índices de avaliação que frequentemente são utilizados com base na concorrência (MASCARENHAS; MANZINI, 2008).

Os índices de absenteísmo, por exemplo, permitem estimar perdas relacionadas às ausências dos funcionários no ambiente de trabalho (MASCARENHAS; MANZINI, 2008). Entretanto, acredita-se que estes indicadores são insuficientes, uma vez que não contem- 
plam as dimensões da GP ao desempenho das organizações, ou seja, não são relacionadas à eficácia das práticas de Gestão de Pessoas e, sim, refletem resultados da eficácia dos seus processos (MASCARENHAS; MANZINI, 2008). Neste sentido, torna-se necessário a utilização de mecanismos de avaliação mais amplos que estejam associados ao desempenho organizacional.

Assim, empresas passaram a utilizar ferramentas ou sistemas de avaliação e mensuração que são associados ao conceito de alinhamento estratégico. Dentre os mais citadas está o Balanced Scorecard (BSC) aplicado à GP (MASCARENHAS; MANZINI, 2008) O BSC, através da utilização de diferentes indicadores, funciona como um sistema que auxilia o processo de alinhamento e mensuração e avaliação de resultados em GP (MASCARENHAS; MANZINI, 2008).

\section{MÉTOdo}

Tratou-se de uma revisão bibliométrica (GIL, 2002) sobre avaliação e mensuração de resultados em GP, organizada em dois momentos. Primeiro, foi realizada uma análise bibliométrica com abordagem quantitativa descritiva. As pesquisas bibliométricas são utilizadas para estudar os aspectos quantitativos da produção e disseminação acadêmica (MACIAS-CHAPULA, 1998) e para avaliação das características das pesquisas, autores, classificação das instituições, dentre outros (HAYASHI et al., 2005; VIEIRA, 2007). Num segundo momento, foi realizada a análise qualitativa, de modo a analisar o conteúdo veiculado nas publicações.

A pesquisa foi realizada nos seguintes bancos de dados eletrônicos: Scientific Periodicals Electronic Library (SPELL), Scientific Electronic Library Online (SciELO) e EBSCO (esta com acesso às bases de dados Business Source Premier, Fonte Acadêmica, Academic Search Ultimate, MEDLINE Complete, Abstracts in Social Gerontology, Art \& Architecture Source, Dentistry \& Oral Sciences Source, Regional Business News, World Politics Review). A pesquisa, conduzida no mês de julho de 2017 utilizou como estratégias de buscas a combinação das seguintes palavras-chave presentes em artigos e livros de referência: "Recursos Humanos AND Resultados"; "Gestão de Pessoas AND Resultados"; "Gestão de Pessoas AND Avaliação"; “Recursos Humanos AND Avaliação"; "Recursos Humanos AND Mensuração"; 
"Gestão de Pessoas AND Mensuração"; "Gestão de Pessoas AND Indicadores"; "Recursos Humanos AND Indicadores"; "Recursos Humanos AND Impactos"; "Gestão de Pessoas AND Impactos"; "Recursos Humanos AND Performance"; Gestão de Pessoas AND Performance".

A busca avançada foi feita nos campos mais amplos dos motores de busca de cada base. Foram utilizados como critérios de inclusão: artigos científicos completos e artigos de revisão completos publicados em qualquer tempo, publicados em periódicos brasileiros e vinculados ao país de origem Brasil. Foram usados, como critérios de exclusão: artigos em duplicata, que não tratassem do tema e textos em formato de monografias, dissertações, teses e livros ou outros. Excluíram-se os artigos que tratavam de avaliação de desempenho dos indivíduos.

Após a aplicação destes critérios, os artigos foram primeiramente selecionados em função dos seus títulos e, depois, pela leitura de seus respectivos resumos. Por fim, os artigos incluídos tiveram seu texto completo analisado a partir dos critérios de elegibilidade, relacionados aos objetivos do estudo. Foi elaborado um quadro de análise para a análise bibliométrica. Depois, foram definidas as categorias de análise: 1) total de artigos; 2) cronologia das publicações; 3) natureza das publicações; 4) periódicos das publicações; 5) instituições das publicações; 6) autores e coautores das publicações; 7) indicadores de RH utilizados nas publicações; 8) análise dos títulos das publicações; 9) cenários dos estudos publicados 10) contribuições/ avanços na temática; 11) entraves ao processo. Tratou-se, portanto, de análise com abordagem predominantemente descritiva (RUDIO, 2014). A análise bibliométrica foi organizada por dois avaliadores, os quais realizaram três etapas de análise: individual, comparativa e conclusiva sobre a definição dos artigos que integram este estudo.

\section{Resultados}

\subsection{Total de artigos}

Foram encontrados os seguintes achados: na SPELL, quarenta e nove artigos (49); na SciELO, novecentos e nove (909); e, na EBS$\mathrm{CO}$, oitenta e sete (87). No total, foram encontrados mil e quarenta 
e cinco artigos (1045). Foram excluídos sessenta e nove (69) artigos repetidos e novecentos e quarenta e dois (942) artigos que não possuíam como foco central o tema em questão. Assim, a análise final contou com 34 artigos.

\subsection{Cronologia das publicações}

Identificar a evolução da publicação nas temáticas ao longo do período foi o que motivou a construção desse tópico. Para tanto, a análise das publicações por ano foi organizada em triênios. Observou-se as publicações tiveram concentração percentual mais elevada nos triênios 2006-2008 (24\%, 8 artigos), 2009-2011 (21\%, 7 artigos), 2012-2014 (18\%, 6 artigos), e 2015-2017 (24\%, 8 artigos). O triênio com o menor número de publicações compreendeu os anos entre 2000 e 2002 ( $3 \%$, equivalente a 1 artigo).

Tabela 1 - Percentual de publicações na área por triênio (2000 a 2017)

\begin{tabular}{lll}
\hline Triênio & Número absoluto & Percentual (\%) \\
\hline $2000-2002$ & 1 & $3 \%$ \\
$2003-2005$ & 4 & $12 \%$ \\
$2006-2008$ & 8 & $24 \%$ \\
$2009-2011$ & 7 & $21 \%$ \\
$2012-2014$ & 6 & $18 \%$ \\
$2015-2017$ & 8 & $24 \%$ \\
TOTAL & 34 & $100 \%$ \\
\hline
\end{tabular}

Fonte: dados da pesquisa (2017)

Entende-se que esse crescimento nas publicações tenha se dado especialmente após 2006 em função do apontado por Lengnick-Hall et al. (2009). Há de se considerar, então, que o tema Gestão Estratégica passou por diversos momentos, iniciando pelo próprio entendimento do que seria estratégico, para então passar pelos estudos sobre ajustamento e necessidade de alinhamento externo e interno. Necessidade de considerar os diferentes contextos (indústria, serviços, etc.) vieram na sequência dos estudos, para então se centrarem na contribuição estratégica da área. Novos estudos vieram sobre 
sistemas de GP e estruturas, atuação em cadeias e contextos internacionais, bem como estudos sobre a implementação e execução da GEP. Só então vieram os estudos sobre a mensuração dos resultados de GP. Os autores abordam o contexto internacional de publicações, bem como o contexto internacional que as transformações de GP aconteceram. No Brasil, elas são mais tardias e enfrentam diferentes desafios, tal como situado no referencial teórico.

Outro ponto que ajuda a discutir os resultados encontrados estaria na possível maior demora na publicação de artigos em revistas. Vasquez et al. (2013) discutem a partir de uma meta-análise que as publicações sobre desempenho (individual ou organizacional) tiveram um pico entre os anos de 2002 e 2005, e uma considerável queda a partir de 2006 . Os artigos sobre o tema (91\% do encontrado) foram publicados no Enanpad, eventos onde a discussão e publicação de artigos tem maior abrangência e dinamicidade.

\subsection{Natureza das publicações}

Procurando-se verificar o contexto da pesquisa, investigou-se a natureza da pesquisa aplicada pela classificação: quantitativa, qualitativa e mista ("quali-quanti"). Assim, quanto à natureza das publicações, ou seja, ao método de abordagem de pesquisa observou-se que 59\% utilizaram abordagem qualitativa (20 artigos), 12\% (quatro artigos) são de natureza quantitativa e 18\% (seis artigos) são de natureza mista (Figura 2). Quatro artigos (11\%) são teóricos.

Figura 2 - Natureza das publicações

Fonte: dados da pesquisa (2017)

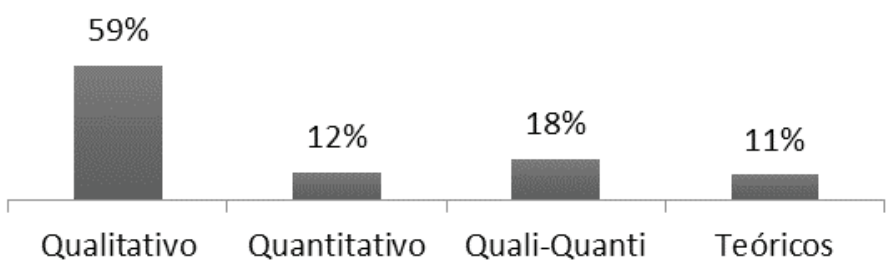

Na mesma linha de análise proposta acima quanto aos desdobramentos do tema GEP ao longo dos tempos (LENGNICK-HALL 
et al., 2009), entende-se haver um movimento inicial pela busca por maior entendimento do que estaria sendo pensado e conduzido nas empresas. Nesse sentido, estudos qualitativos permitiriam uma maior compreensão do panorama geral. Os estudos quanti já estão mais voltados para o teste de modelos e variáveis.

\subsection{Periódicos das publicações}

Buscou-se, aqui, identificar percentualmente a distribuição da publicação dos artigos nas revistas. Vinte e um periódicos foram responsáveis pela publicação dos artigos analisados, com distribuição diversificada entre aqueles que mais publicaram. Observou-se que a média de publicações do tema analisado nos periódicos foi de 2,43 artigos anualmente. Entretanto, a maioria dos periódicos 12 (34\%) publicou somente um artigo.

A maior concentração de publicações (4) esteve na Revista Escola de Enfermagem da Universidade de São Paulo (USP), 12\% do total de publicações. A Revista Latino-Americana de Enfermagem e a revista Acta Paulista de Enfermagem publicaram 4 artigos (9\%). As demais publicaram 2 artigos (6\%) (Tabela 2). Uma observação pertinente a se fazer é o que fato que os artigos publicados nessas revistas, em sua maioria, apresentam estudos que empregam, geralmente, indicadores de GP mais tradicionais, como índices para o cálculo de absenteísmo, de rotatividade, índices de satisfação com o trabalho e aplicação do Balanced Scorecard (BSC).

Tabela 2 - Publicações por periódico

\begin{tabular}{|c|c|c|}
\hline Periódico & $\begin{array}{l}\text { Total de } \\
\text { publicações }\end{array}$ & $\%$ \\
\hline Revista Escola de Enfermagem USP & 4 & 12 \\
\hline Revista Latino-Americana de Enfermagem & 3 & 9 \\
\hline Acta Paulista de Enfermagem & 3 & 9 \\
\hline Revista PRETEXTO & 2 & 6 \\
\hline Revista de Gestão (REGE-USP) & 2 & 6 \\
\hline Revista de Administração e Contabilidade da Unisinos (BASE) & 2 & 6 \\
\hline Revista de Administração de Empresas & 2 & 6 \\
\hline Organizações \& Sociedade & 2 & 6 \\
\hline Revista de Administração Pública (RAP) & 2 & 6 \\
\hline Outros & 12 & 34 \\
\hline TOTAL & 34 & 100 \\
\hline
\end{tabular}

Fonte: dados da pesquisa (2017) 


\subsection{Instituições das publicações}

As Instituições de Ensino com maior número de publicações foram a Universidade de São Paulo (USP) com 9 (26\%), Fundação Getúlio Vargas (FGV) e Universidade Federal de Minas Gerais (UFMG) com 3 cada (9\%), Faculdade de Medicina de São José do Rio Preto (FAMERP), Fundação Universidade Regional de Blumenau (FURB) e a Universidade do Sul de Santa Catarina (UNISUL) com 2 (6\%). As demais Instituições de Ensino publicaram juntas 13 artigos (38\%) (Tabela 3).

Tabela 3 - Publicações por Instituições de Ensino

\begin{tabular}{lll}
\hline Universidade & Quantidade & Percentual \\
\hline USP & 9 & $26 \%$ \\
FGV & 3 & $9 \%$ \\
UFMG & 3 & $9 \%$ \\
FAMERP & 2 & $6 \%$ \\
FURB & 2 & $6 \%$ \\
UNISUL & 2 & $6 \%$ \\
Outras & 13 & $38 \%$ \\
TOTAL & 34 & $\mathbf{1 0 0} \%$ \\
\hline
\end{tabular}

Fonte: dados da pesquisa (2017)

\subsection{Autores e coautores das publicações}

Objetivou-se aqui identificar possíveis concentrações de autores nas publicações. A análise das publicações por autores e coautores possibilitou identificar o envolvimento entre 32 universidades e 104 pesquisadores. Destacaram-se seis Instituições de Ensino concentrando a maior quantidade de pesquisadores: USP (Universidade de São Paulo - com 25\%, 26 pesquisadores), FGV (Fundação Getúlio Vargas - com 9\%, 9 pesquisadores), PUC-PR (Pontifícia Universidade Católica do Paraná - 6 pesquisadores), UFMG (Universidade Federal de Minas Gerais - 6 pesquisadores). Em média as instituições apresentam 9,36 pesquisadores, entre autores e coautores (Figura 3). 
Figura 3 - Representação das instituições por autores e coautores

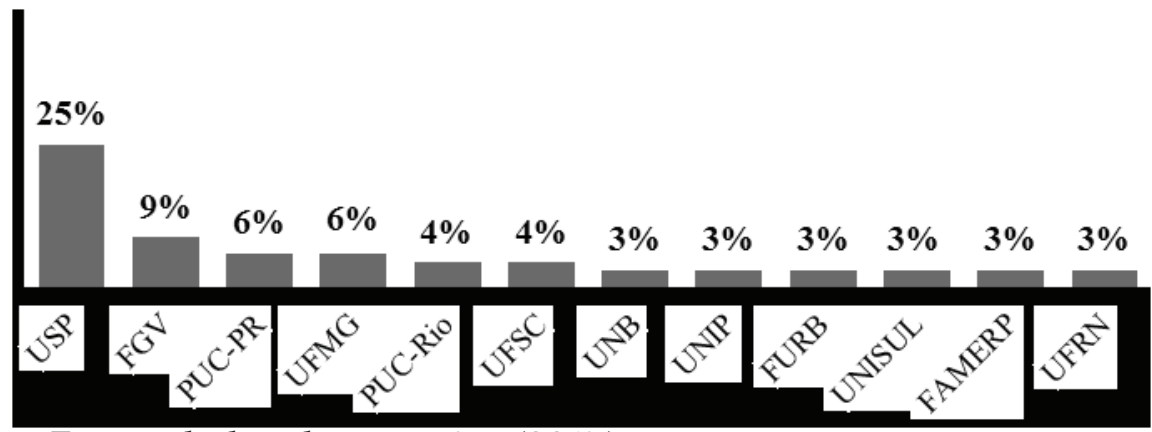

Fonte: dados da pesquisa (2017)

Percebe-se haver uma pulverização de autores, os quais produziram apenas um (1) artigo no tema avaliação e mensuração de resultados em Gestão de Pessoas. Somente Paulina Kurcgant publicou três artigos como primeira autora. Em relação aos coautores, retirando-se os autores Lindolfo Galvão de Albuquerque (2 artigos), Márcia Gallan Perroca (2 artigos), Valéria Castilho (2 artigos), Daisy M. Rizatto Tronchin (2 artigos) e Marta Maria Melleiro (3 artigos), os demais coautores participaram em somente uma publicação.

\subsection{Mecanismos/Indicadores de Gestão de Pessoas utiliza- dos nas publicações}

Os mecanismos ou indicadores tradicionais de avaliação de GP abordados nos artigos foram: Balanced Scorecard (24\%, aparecendo em 8 artigos), Absenteísmo (21\%, 7 artigos), Clima organizacional ( $9 \%, 3$ trabalhos) e Rotatividade (3\%, 1 publicação). Apenas 1 artigo abordou indicadores operacionais de pessoas, como média de salários de funcionários, custo de supervisão, indicador de estrutura de pessoal, de recrutamento por vaga, de investimento por empregado e indicador de antiguidade relativa.

Todavia, $41 \%$ das publicações analisadas (14 artigos) apresentaram outros sistemas e indicadores personalizados à estrutura de pessoal do cenário analisado. Exemplos desses indicadores foram Skandia Navigator, Data Envelopment Analysis, horas de treinamento e-learning, sinistralidade de assistência médica e taxa de ocupação hoteleira. 


\subsection{Análise dos títulos das publicações}

Essa parte do trabalho buscou descrever, a partir dos títulos, o quê o artigo apresenta como propósito central de investigação. Para tanto, a análise dos títulos dos artigos foi organizada em seis eixos temáticos (Tabela 4). A maioria dos artigos buscou abordar o alinhamento entre a GP e a estratégia da organização (35\%, 12 publicações). Em seguida, está o absenteísmo e a avaliação e mensuração de resultados, ambos com $21 \%$ (7 publicações).

Tabela 4 - Análise dos títulos das publicações

\begin{tabular}{lll}
\hline Expressões-chave dos títulos & $\begin{array}{l}\text { No de } \\
\text { repetições }\end{array}$ & $\begin{array}{l}\text { Percen- } \\
\text { tual (\%) }\end{array}$ \\
\hline $\begin{array}{l}\text { Indicadores e medidas de desempenho } \\
\text { para o alinhamento estratégico }\end{array}$ & 12 publicações & $35 \%$ \\
$\begin{array}{l}\text { Absenteísmo } \\
\text { Avaliação e mensuração de resultados }\end{array}$ & 7 publicações & $21 \%$ \\
Balanced ScoreCard (BSC) & 4 publicações & $21 \%$ \\
Rotatividades & $12 \%$ \\
Clima Organizacional & 2 publicações & $6 \%$ \\
\hline
\end{tabular}

Fonte: dados da pesquisa (2017)

\subsection{Cenários dos estudos publicados}

A análise dos cenários - ambientes em que os estudos foram realizados - das publicações identificou que 35\% dos trabalhos (12 artigos) foram executados na área da Saúde, mais precisamente em hospitais de ensino, e em relação aos profissionais, com as equipes de enfermagem. Em contrapartida, 47\% do total de publicações (16 artigos) não esclarecem o cenário pesquisado, apresentam apenas as abordagens teóricas utilizadas, mais precisamente, estudos que se concentram na descrição de indicadores de Gestão de Pessoas (Tabela 5). 
Tabela 5 - Cenários de realização dos estudos

\begin{tabular}{lll}
\hline Cenários & $\mathrm{n}$ & $\%$ \\
\hline Saúde (equipes de trabalho, hospitais) & 12 & 35 \\
Educação (instituições de ensino) & 2 & 6 \\
Indústria (setores) & 1 & 3 \\
Justiça & 1 & 3 \\
Serviços (hotelaria) & 2 & 6 \\
Não apresentam o local pesquisado & 16 & 47 \\
\hline TOTAL & $\mathbf{3 4}$ & $\mathbf{1 0 0 \%}$ \\
\hline
\end{tabular}

Fonte: dados da pesquisa (2017)

Como já mencionado, além dessa etapa quantitativa sobre a produção científica acerca da avaliação e mensuração de resultados em GP, a segunda etapa (de cunho qualitativo) buscou investigar quais foram as contribuições e os entraves identificados, em estudos aplicados ou testados nas realidades organizacionais que serviram de estrutura para as pesquisas. Assim, os pontos 4.10 (contribuições) e 4.11 (entraves) foram organizados a partir das sínteses conclusivas presentes nos artigos abordados neste estudo.

\subsection{Contribuições/Avanços na temática}

A análise da produção científica possibilitou identificar vários pontos julgados como positivos ao utilizar indicadores para avaliação e mensuração dos resultados em GP. Grande parte dos artigos buscou centralizar as pesquisas no relacionamento entre a área de GP e o desempenho organizacional (BARRETO; ALBUQUERQUE; MEDEIROS, 2014; CESAR, 2015; FERNANDES; FLEURY; MILLS, 2006; GONÇALVES; TORTOLI, 2015; NASCIMENTO et al., 2010; OLIVEIRA; OLIVEIRA, 2011; RODRIGUES; TORRES, 2015; SILVEIRA; FILHO, 2013; SOARES; MAZON, 2016) trazem a discussão sobre o que seria desempenho organizacional para então pensar no desempenho envolvendo GP. Os autores citam Ferris et al. (1998) ao apontar que desempenho organizacional é um construto multidimensional que necessita de múltiplos critérios de efetividade em função dos elementos internos e externos pelos quais as 
organizações são responsáveis. Quatro tipos de resultados seriam possíveis: resultados de GP, resultados organizacionais, resultados financeiros e resultados de mercado de capital, que devem ser tomados em conjunto. Segundo Oliveira e Oliveira (2011), os de GP constituem consequências sobre as competências dos empregados individualmente; os organizacionais constituem resultados de GP sobre o conjunto de competências dos empregados transformados em competências organizacionais; os resultados financeiros ou contábeis, por sua vez, são as consequências das práticas sobre índices financeiros e, por fim, os resultados de mercado de capital são as consequências das práticas de GP sobre a valorização da organização no mercado financeiro (OLIVEIRA; OLIVEIRA, 2011, p 654). Os autores salientam que as chamadas práticas de alto desempenho ou de alto comprometimento são apontadas como promotoras de um maior desempenho, na medida que podem agregar valor organizacional devido aos seus processos e estruturas únicas. A relação positiva entre GP e desempenho organizacional se amplia quando as práticas de GP são combinadas em um sistema ou dois ou mais resultados de desempenho organizacional são levados em consideração (OLIVEIRA; OLIVEIRA, 2011).

As medidas de desempenho econômico-financeiro foram destacadas em Da Silva, Basques e Facco (2006), em Piellusch e Taschner (2009) e em Corso et al. (2014). O uso dos indicadores relacionado às políticas e práticas de GP e a associação das ações de GP aos resultados organizacionais destacadas por Lacombe e Albuquerque (2008) e a relação de GP com os stakeholders foi discutida em Tinoco (2003).

Nos artigos, foi possível identificar que a mensuração dos resultados em GP auxilia a reduzir as críticas sobre seu carácter subjetivo (SILVEIRA; FILHO, 2013) e assim proporcionar que a GP seja melhor reconhecida como estratégica (BARRETO; ALBUQUERQUE; MEDEIROS, 2014). Esses últimos autores trazem à discussão as relações entre as configurações de estratégias de GP, as capacidades organizacionais e o desempenho organizacional no contexto de hotéis. Destaca-se, também, a divulgação dos indicadores de GP como mecanismo de fortalecimento da imagem positiva da empresa, para possibilitar o engajamento dos funcionários e aumentar o senso de comprometimento do empregado (CESAR, 2015). 
Foi possível observar o uso de indicadores tradicionais como absenteísmo, rotatividade, acidentes de trabalho, quadro de pessoal, horas de treinamento relacionados ao desempenho hospitalar (RODRIGUES; TORRES, 2015). Eles foram considerados bons indicadores (KURGANCT; MELLEIRO; TRONCHIN, 2008), pois possibilitam conhecer a realidade institucional (SANCINETTI et al., 2011) através da identificação de doenças ocupacionais, insatisfação dos colaboradores com as condições institucionais e relacionamento interpessoal inadequado (KURCGANT et al., 2015), problemas relacionados à cultura organizacional, saúde e bem estar dos trabalhadores e taxas de ausências não previstas (BARGAS; MONTEIRO, 2014); (FAKIH; TANAKA; CARMAGNANI, 2009). Tais constatações são importantes para o gerenciamento do custo de rotatividade de pessoal e o impacto financeiro do custo de desligamento dos colaboradores (RUIZ; PERROCA; JERICÓ, 2016), contribuindo para o alcance da qualidade na prestação dos serviços (KURCGANT et al., 2009). Propõem a utilização de indicadores de qualidade como subsídio a avaliação em GP (KURCGANT et al., 2009) na área de saúde e a visão das relações sistêmicas entre tais indicadores e os processos de GP (RUIZ; PERROCA; JERICÓ, 2016).

Os indicadores de GP permitem analisar resultados de competência e desempenho através de satisfação dos funcionários, nível de competência profissional, processos internos, clientes e demonstrativos financeiros (FERNANDES; FLEURY; MILLS, 2006; VIEIRA; FILENGA, 2012), possibilitam a reflexão sobre a condução do processo admissional, a alocação de recursos, a redução de custos e qualidade dos processos (OKANO; CASTILHO, 2007). O efeito sinérgico entre as práticas de gestão de pessoas tomadas como interdependentes e as melhorias gerais no desempenho dos empreendimentos são também apontadas (BARRETO; ALBUQUERQUE; MEDEIROS, 2014).

Os indicadores multicritérios foram considerados importantes uma vez que contribuem para a gestão de fatores econômico-financeiros e não financeiros (GONÇALVES; TORTOLI, 2015). Assim, a análise da produção científica evidenciou maior destaque para alguns destes sistemas, como o BSC e o Skandia Navigator.

O BSC foi apontado por possibilitar a integração de objetivos organizacionais e individuais, melhorias no alcance de metas e na 
qualidade dos serviços oferecidos (MALACARNE; BALASSIANO; NOSSA, 2016) e a tradução das estratégias organizacionais através de objetivos indicadores e iniciativas (ROCHA-PINTO; DEL CARPIO, 2011). Proporciona uma visão holística da organização através de medidas tradicionais de desempenho financeiro e medidas não financeiras (CORSO et al., 2014) (LIMA; SOARES; LIMA, 2011), contribuindo para o alinhamento organizacional (GALAS; PONTE, 2005). O uso do Skandia Navigator proporciona foco financeiro (passado), foco nos clientes, nos recursos humanos e nos processos (presente) e foco na capacidade de renovação e desenvolvimento organizacional (futuro) (SOARES; MAZON, 2016).

\subsection{Entraves ao processo}

A análise da produção científica brasileira sobre avaliação e mensuração de resultados em GP também apontou alguns pontos negativos ou que ainda precisam ser mais explorados. Parece haver uma diferenciação pouco clara do que seriam os resultados de GP e a contribuição indireta das ações de GP nos resultados organizacionais. Como são muitos os resultados de GP que se procura obter, torna-se difícil o detalhamento do fluxo causal entre ações de GP e resultados (LACOMBE; ALBUQUERQUE, 2008).

Existem empresas que utilizam os indicadores de GP apenas de maneira operacional, apresentando apenas resultados da própria área, não acompanhando o cumprimento o papel estratégico (PIELLUSCH; TASCHNER, 2009). Os indicadores de absenteísmo na área de saúde são limitados e necessitam de criação de banco de dados para acompanhar a evolução dos problemas identificados, realizar as correções necessárias e avaliar os resultados (SILVA; MARZIALE, 2000).

Existem, também, desafios para criar indicadores para a avaliação da taxa de satisfação no trabalho e políticas de remuneração (KURGANCT; MELLEIRO; TRONCHIN, 2008). Alguns executivos desconhecem indicadores de resultados para ações e programas de qualidade de vida, ou desconhecem tais resultados (OLIVEIRA; LIMONGI-FRANÇA, 2005). Há a necessidade de se difundir um modelo de avaliação e mensuração dos resultados de Qualidade de Vida no Trabalho (OLIVEIRA; LIMONGI-FRANÇA, 2005). Muitas 
métricas são de ordem operacional e financeira somente (OLIVEIRA; LIMONGI-FRANÇA, 2005).

O BSC pode representar uma ameaça através da maior exposição das áreas e das pessoas (ROCHA-PINTO; DEL CARPIO, 2011). A adequação do BSC para Instituições de Ensino Superior parece ser um desafio, uma vez que o BSC é efeito do planejamento estratégico (LIMA; SOARES; LIMA, 2011). Ainda existem divergências entre o objetivo de avaliação e a ferramenta escolhida (NASCIMENTO et al., 2010).

Chama a atenção a baixa divulgação de indicadores de GP, especialmente relacionadas a planos de remuneração variável, planejamento de carreira e recrutamento e seleção e baixa evidenciação de indicadores relacionados à saúde e bem-estar dos funcionários (CESAR, 2015). A autora destaca ainda que embora o capital humano seja apontado como uma das principais fontes de competitividade, ainda não há medidas que elucidem melhor a relação entre as pessoas e o resultado econômico-financeiro das organizações.

A superioridade de sistemas com conjuntos de indicadores financeiros e não financeiros ainda é difícil de ser comprovada com rigor empírico (GONÇALVES; TORTOLI, 2015). Os indicadores qualitativos devem ser mais aprofundados para que possam gerar uma visão mais abrangente dos sistemas em gestão de pessoas (KURCGANT et al., 2009). De modo geral, assim, os artigos sinalizam que há muito campo para estudos.

\section{Discussão dos ACHADOS}

A produção científica brasileira acerca dos indicadores de avaliação e mensuração de resultados em Gestão de Pessoas revelam a busca pelo aprofundamento da contribuição acadêmica para as práticas em GP do mundo empresarial. De fato, pesquisas que evidenciem a realidade das organizações, em seus mais diversos contextos e estruturas, se fazem fundamentais para a garantia do avanço dos métodos de trabalho, assim como para a manutenção dos negócios e do bem-estar social nas empresas.

Nesse artigo, observou-se alguns momentos em termos de pesquisas: em um dos primeiros momentos de estudo em relação à temática, estaria a busca por evidenciar a realidade das organizações, 
as transformações ocorridas e as esperadas da área, e apresentar de que forma pode-se estar direcionando uma atuação mais estratégica. Nesse sentido, os estudos destacam a necessidade de desenhar um modelo de avaliação dos resultados em GP.

De modo geral, como um segundo momento, tem-se a discussão sobre o alinhamento das práticas de GP e a mensuração dos resultados. Associação entre temas como competências, inovação, competitividade e humanização foram identificados. Em contextos mais específicos, como a de saúde, observa-se um movimento voltado para a qualidade, buscando-se relacionar os indicadores de qualidade ao gerenciamento em GP; a preocupação com absenteísmo e rotatividade, investigando, no primeiro caso, as causas que levam os profissionais da saúde a essa prática e o posicionamento estratégico dos gestores dessas equipes; subprocessos envolvidos na rotatividade e os custos foram julgados como importantes aspectos a serem investigaodos. O índice de clima organizacional foi trabalho sob a ótica da concepção de uma metodologia de diagnóstico e a influência dos perfis de profissionais de Enfermagem.

Mais recentemente, pode-se sinalizar, ainda dentro da busca por esse alinhamento estratégico, a proposição de modelos que possam clarificar as relações entre desempenho, o que inclui desempenho econômico financeiro, e as práticas de GP. Temas como capacidades organizacionais e recursos são trazidos nos artigos.

Assim, entende-se, assim como já apontava Legge (2005), e que continua sendo válido, serem necessários ainda esforços a respeito de como conceituar desempenho e como estabelecer uma ligação entre GP e desempenho organizacional, sendo que esse ponto precisa ser ainda melhor desbravado. Um dos caminhos apontados pela autora seria a do desenvolvimento de modelos que incluam variáveis intervenientes chave. Observam-se já alguns estudos nesse sentido, trazendo novos modelos e ainda testando variáveis e relações. Sobretudo, aponta-se a necessidade ainda de maior criticidade em relação à temática no que se refere à constituição e aplicabilidade de modelos, sistemas e indicadores de GP no ambiente organizacional contemporâneo. 


\section{CONSIDERAÇõES FINAIS}

As mudanças sociais, empresariais e estruturais constituídas no âmbito do trabalho (legislação, novos modelos empresariais e as distintas relações empresariais com os trabalhadores) vem demandando uma reorganização que se consolidou externa (as trocas comerciais, as relações de mercado e a composição de novos sistemas de produção) e internamente (as estratégias de negócios, as políticas e práticas de Gestão de Pessoas e a reorganização do sistema produtivo).

É fato, portanto, que a área de Gestão de Pessoas nas organizações, nas últimas décadas, vem atravessando uma série de mudanças em decorrência da dinâmica do mercado e das organizações em seus contextos, principalmente quanto à capacidade de articulação da área com os objetivos e estratégia organizacionais, algo que impacta no papel que desempenha e protagoniza no interior das organizações. Nesse sentido, sua elevação a um nível estratégico, assim como sinalizaram os estudos aqui investigados, mobilizou gradualmente, em um primeiro momento, o entendimento de seu caráter estratégico; posteriormente, sua relação e alinhamento com os ambientes interno e externo, em que pese os diferentes campos - tais como indústria, saúde, educação e comércio -, o cenário internacional, a estruturação da GP e sua implementação e execução.

Reforçando esse olhar, os artigos apontaram para um estreitamento da Gestão de Pessoas com o desempenho organizacional, enfatizando a necessidade de sinergia com os resultados organizacionais, financeiros e do mercado de capital. Numa tentativa de desenvolver as competências organizacionais a partir da Gestão de Pessoas, notou-se a partir dos estudos a relevância da consolidação e do desenvolvimento de competências individuais. Tal posicionamento possui como fundamento práticas de GP capazes de garantir práticas de altos desempenho e comprometimento, bem como práticas que agreguem valor à estrutura organizacional como um todo.

Associado a isso, os estudos também sinalizaram diferentes indicadores de mensuração de resultados em Gestão de Pessoas, como absenteísmo, rotatividade, acidentes de trabalho, horas de treinamento e saúde e bem-estar de trabalhadores. Entretanto, ergue-se, conforme os estudos, uma crítica ao uso operacional dos indi- 
cadores, o que contrapõe o papel estratégico que vem se buscando à área, visto que os indicadores ainda permanecem sendo aplicados na mensuração de resultados das próprias áreas em grande parte das organizações.

Quanto à proposta de pesquisa deste estudo argumenta-se que a pesquisa bibliométrica acerca da produção científica brasileira sobre avaliação e mensuração de resultados em GP identificou incremento nas publicações ao longo dos anos, predominância de pesquisas qualitativas e distribuição diversificada das publicações entre instituições de ensino, autores e coautores brasileiros. Constatou-se o uso de diferentes indicadores, com destaque para o Balanced Scorecard e Skandia Navigator, além de outros destinados à absenteísmo, clima organizacional e rotatividade. Houve predomínio de contextos ligados à saúde. A mensuração de resultados de GP fomenta uma visão estratégica dessa gestão, elevando seu status e reduzindo críticas sobre seu caráter subjetivo.

Este artigo contribui para os estudos sobre GEP, uma vez que apresenta um panorama atual das pesquisas nacionais sobre o tema em revistas científicas. Destaca-se que o estudo não apresentou quaisquer limitadores de tempo para a seleção dos artigos e utilizou diversos cruzamentos de palavras-chave, possibilitando maior número de publicações sobre esta temática. Como limitações, destaca-se a restrição da pesquisa a três bases de dados, o fato de não ter incluído eventos científicos. Em função disso, estimula-se o desenvolvimento de novas pesquisas sobre a temática em diversos formatos.

Como principais temas de pesquisas futuras sugere-se a análise do cenário internacional, o desenvolvimento de pesquisas empíricas em organizações nacionais e internacionais, a ampliação e diversificação dos tipos de organizações (por exemplo, organizações sem fins lucrativos, ONGs e organizações públicas). Por fim, entende-se que deve existir um amplo debate entre pesquisadores e gestores sobre a relevância e a emergência do tema, especialmente em tempos de crise. 


\section{REFERÊNCIAS}

ALBUQUERQUE, Lindolfo Galvão de. Estratégias de recursos humanos e competitividade. Administração contemporânea: perspectivas e estratégias. São Paulo: Atlas, p. 215-238, 1999.

ALBUQUERQUE, Lindolfo Galvão de. A gestão estratégica de pessoas. In: PROGEP. As pessoas na organização. São Paulo: Gente, 2002.

ALMEIDA, Martinho Isnard Ribeiro De; TEIXEIRA, Maria Luiza Mendes; MARTINELLI, Dante Pinheiro. Por que administrar estratégicamente recursos humanos? Revista de Administração de Empresas, [s. 1.], v. 33, n. 2, p. 12-24, 1993.

ASHTON, Chris; HAFFENDEN, Mike; LAMBERT, Andrew. The "fit for purpose" HR function. Strategic HR Review, [s. 1.], v. 4, n. 1, p. 32-35, 2004.

ASSIS, Marcelino Tadeu de. Indicadores de gestão de recursos humanos: usando indicadores demográficos, financeiros e de processos na gestão do capital humano. Rio de Janeiro: Qualitymark, p. 48, 2005.

BARGAS, Eliete Boaventura; MONTEIRO, Maria Inês. Fatores relacionados ao absenteísmo por doença entre trabalhadores de Enfermagem. ACTA Paulista de Enfermagem, [s. 1.], v. 27 , n. 6 , p. 533-538, 2014.

BARRETO, Leiliane Michelle Trindade da Silva; ALBUQUERQUE, Lindolfo Galvão De; MEDEIROS, Carlos Alberto Freire. Estratégias de gestão de pessoas e desempenho organizacional na hotelaria: o papel das capacidades organizacionais. Revista Brasileira de Pesquisa em Turismo, [s. 1.], v. 8, n. 2, p. 340-360, 2014.

BECKER, Brian E.; ULRICH, Dave; HUSELID, Mark A. Gestão estratégica de pessoas com scorecard. Elsevier Brasil, 2001.

BIANCHI, Eliane Maria Pires Giavina; QUISHIDA, Alessandra; FORONI, Paula Gabriela. Atuação do líder na gestão estratégica de pessoas: reflexões, lacunas e oportunidades. Revista de Administração Contemporânea, [s. 1.], v. 21, n. 1, p. 41-61, 2017.

BOXALL, Peter; PURCELL, John. Strategy and Human Resource Management. New York: Palgrave, 2003.

CANÇADO, Vera L.; VENDRAMINE, Maraí; OLIVEIRA, Elizângela de Jesus. Revisitando as Quatro Faces da Gestão de Recursos Humanos: estudo em empresas no Brasil. Navus Revista de Gestão e Tecnologia, [S.1.], v. 10, p. 01-14, 2020.

CASTRO, José Márcio De; SILVA, Clara Luisa Oliveira. A influência da cultura organizacional e da orientação estratégica na gestão de recursos humanos: um estudo de caso. Revista de Administração da UNIMEP, [s. 1.], v. 10, n. 1, p. 1-28, 2012.

CESAR, Ana Maria Roux V. C. Medidas de desempenho da área de recursos humanos e seu relacionamento com indicadores de desempenho econômico. Revista de Gestão, [s. 1.], v. 22, n. 1, p. 77-94, 2015.

CHAHAL, Hardeep; JYOTI, Jeevan; RANI, Asha. The effect of perceived high-performance human resource práctices on business performance: role of organizational learning. Global Business Review, [s. 1.], v. 17, n. 3, p. 107-132, 2016. 
COMBS, James; LIU, Yongmei; HALL, Angela; KETCHEN, David. How much do high-performance work practices matter? A meta-analysis of their effects on organizational performance. Personnel psychology, v. 59, n. 3, p. 501-528, 2006.

CORSO, Jansen Maia Del; PETRASKI, Susan Jareck; SILVA, Wesley Vieira da; TAFFAREL, Marinês. Gestão estratégica de recursos humanos: identificando o processo de alinhamento estratégico. Tourism \& Management Studies, [s. 1.], v. 10, n. 1, p. 49-57, 2014.

FAKIH, Flávio Trevisan; TANAKA, Luiza Hiromi; CARMAGNANI, Maria Isabel Sampaio. Ausências dos colaboradores de enfermagem do pronto-socorro de um hospital universitário. ACTA Paulista de Enfermagem, [s. 1.], v. 25, n. 3, p. 378-385, 2009.

FERNANDES, Bruno Henrique Rocha; FLEURY, Maria Tereza Leme; MILLS, John. Construindo o diálogo entre competência, recursos e desempenho organizacional. Revista de Administração de Empresas, [s. 1.], v. 46, n. 4, p. 48-66, 2006.

FERRIS, Gerald R.; FRINK, Dwight D.; ARTHUR, Michelle M. Toward a social context theory of the human resource management-organization effectiveness relationship. Human resource management review, v. 8, n. 3, p. 235-264, 1998.

GALAS, Eduardo Santos; PONTE, Vera Maria Rodrigues. O Balanced Scorecard e o alinhamento organizacional: um estudo de casos múltiplos. BASE - Revista de Administração e Contabilidade da Unisinos, [s. 1.], v. 2, n. 3, p. 176-188, 2005.

GIL, Antônio Carlos. Como elaborar projetos de pesquisa. 4a ed. São Paulo: Editora Atlas, 2002.

GONÇALVES, Rosana Carmen de Meiroz Grillo; TORTOLI, Julia Peres. Características de indicadores de desempenho em sistemas de incentivo do setor automotivo. Produção Online, [s. 1.], v. 15, n. 3, p. 999-1020, 2015.

HAYASHI, Maria Cristina Piumbato Innocentini; SILVA, Márcia Regina da; HAYASHI, Carlos Roberto Massao; FERREIRA JÚNIOR, Amarílio; FARIA, Leandro Innocentini Lopes de. Competências informacionais para utilização da análise bibliométrica em educação e educação especial especial. Educação Temática Digital, [s. 1.], v. 7, n. 1, p. 11-27, 2005.

HUSELID, Mark a. The impact of human resource management practices on turnover, productivity, and corporate financial performance. The Academy of Management Journal, [s. 1.], v. 38, n. 3, p. 635-672, 1995.

KURCGANT, Paulina; TRONCHIN, Daisy M. Rizatto; MELLEIRO, Marta M.; CASTILHO, Valéria; MACHADO, Valéria Bertonha; PINHEL, Inahiá; SIQUEIRA, Vera Thânia; SILVA, Milena Froes da. Indicadores de qualidade e a avaliação do gerenciamento de recursos humanos em saúde. Revista da Escola de Enfermagem da USP, [s. 1.], v. 43, n. 2, p. 1168-73, 2009.

KURCGANT, Paulina; PASSOS, Andrea Rodrigues; OLIVEIRA, Juliana Macedo Lima de; PEREIRA, Irene Mari; COSTA, Taiza Florêncio. Absenteísmo do pessoal de enfermagem: decisões e ações de enfermeiros gerentes. Revista da Escola de Enfermagem da USP, [s. 1.], v. 49, n. 2, p. 34-40, 2015.

KURGANCT, Paulina; MELLEIRO, Marta Maria; TRONCHIN, Daisy Maria Rizatto. Indicadores para avaliação de qualidade do gerenciamento de recursos humanos em enfermagem. Revista Brasileira de Enfermagem, [s. 1.], v. 61, n. 5, p. 539-544, 2008. 
LACOMBE, Beatriz Maria Braga; ALBUQUERQUE, Lindolfo Galvão De. Avaliação e mensuração de resultados em gestão de pessoas: um estudo com as maiores empresas instaladas no Brasil. Revista de Administração, [s. 1.], v. 43, p. 5-16, 2008.

LACOMBE, Beatriz Maria Braga; CHU, Rebeca Alves. Políticas e práticas de gestão de pessoas: as abordagens estratégica e institucional. Revista de Administração de Empresas, [s. 1.], v. 48, n. 1, p. 25-35, 2008.

LACOMBE, Beatriz Maria Braga; CHU, Rebeca Alves. Políticas e práticas de gestão de pessoas: as abordagens estratégica e institucional. Revista de Administração de Empresas, v. 48, n. 1, p. 25-35, 2008.

LEGGE, Karen. Human Resource Management: rhetorics and realities. New York: Palgrave, 2005.

LEITE, Nildes Raimunda Pitombo; ALBUQUERQUE, Lindolfo Galvão De. Gestão estratégica de pessoas, estratégia de retenção de profissionais e comprometimento organizacional em uma estrutura organizacional remota. Revista de Administração da UFSM, [s. 1.], v. 6, n. 4, p. 803-822, 2013.

LENGNICK-HALL, Mark L.; DRAKE, Brian; LENGNICK-HALL, Cynthia A. Strategic human resource management: The evolution of the field. Human Resource Management Review, [s. 1.], v. 19, n. 2, p. 64-85, 2009.

LIMA, Carlos Rogério Montenegro De; SOARES, Thiago Coelho; LIMA, Mauricio Andrade De. Balanced Scorecard em Instituições de Ensino Superior: uma análise das perspectivas. Estratégias e Negócios, [s. 1.], v. 4, n. 1, p. 183-205, 2011.

MACIAS-CHAPULA, Cesar A. O papel da informetria e da cienciometria e sua perspectiva nacional e internacional. Ciência da Informação, [s. 1.], v. 27, n. 2, p. 134-140, 1998.

MALACARNE, Robson; BALASSIANO, Moisés; NOSSA, Valcemiro. Implicações do BSC nas práticas cotidianas que envolvem a gestão de pessoas: estudo de caso em uma instituição de educação profissional. Organizações \& Sociedade, [s. 1.], v. 23, n. 78, p. 406-420, 2016.

MASCARENHAS, André Ofenhejm; MANZINI, Reinaldo B. Gestão e avaliação de resultados em gestão de pessoas. In: MASCARENHAS, André Ofenhejm et al. (Eds.). Gestão estratégica de pessoas: evolução, teoria e crítica. São Paulo: Cengage Learning, 2008. p. 245-258.

MURITIBA, Patricia Morilha; MURITIBA, Sérgio Nunes; ALBUQUERQUE, Lindolfo Galvão de; JÚNIOR, Flávio Hourneaux. Processo de Avaliação de Resultados de Gestão de Pesssoas: em direção a um modelo. In: XXX ENANPAD 2010, Salvador. Anais... Salvador: ANPAD, 2010.

NASCIMENTO, Sabrina; BORTOLUZZI, Sandro César; COELHO, Ana Lúcia Lima; COELHO, Christiano; ENSSLIN, Sandra Rolim. Ferramentas gerenciais à luz do desempenho organizacional. Revista Pretexto, v. 11, n. 3, 2010.

OKANO, Helena Isuku Horibe; CASTILHO, Valéria. Levantamento do custo do processo admissional de técnico de enfermagem de um hospital de ensino. Revista da Escola de Enfermagem da USP, [s. 1.], v. 41, n. 3, p. 492-499, 2007.

OLIVEIRA, Agostinha Mafalda Barra; OLIVEIRA, Alexandre José. Gestão de Recursos Humanos: uma metanálise de seus efeitos sobre desempenho organizacional. Revista de Administração Contemporânea, [s. 1.], v. 15, n. 4, p. 650-669, 2011. 
OLIVEIRA, Patricia Morilha De; ALBUQUERQUE, Lindolfo Galvão De; MURITIBA, Sérgio Nunes. Uma análise da pesquisa sobre Avaliação de Resultados em Gestão de Pessoas na atualidade. In: XXVII ENANPAD 2003, Atibaia. Anais... Atibaia: ANPAD, 2003.

OLIVEIRA, Patrícia Morilha De; LIMONGI-FRANÇA, Ana Cristina. Avaliação da gestão de programas de qualidade de vida no trabalho. Revista de Administração de Empresas, [s. 1.], v. 4, n. 1, p. 1-21, 2005.

PAROLIN, Sônia Regina Hierro; ALBUQUERQUE, Lindolfo Galvão de Albuquerque. Gestão estratégica de pessoas para a inovação: o caso da Frimesa Cooperativa Central. Revista de Administração da UFSM, [s. 1.], v. 4, n. 1, p. 105-124, 2011.

PAROLIN, Sonia Regina Hierro; DE ALBUQUERQUE, Lindolfo Galvão. Gestão estratégica de pessoas para a inovação: o caso da Frimesa Cooperativa Central. Revista de Administração da UFSM, v. 4, n. 1, p. 105-124, 2011.

PEISENIECE, Līga; VOLKOVA, Tatjana. Necessity to evaluate Human Resource Management in companies of Latvia. Economics \& Management, [s. 1.], v. 15, n. 1, p. 698-704, 2010.

PIELLUSCH, Marcos; TASCHNER, Gisela Black. Indicadores de RH no setor hoteleiro: um estudo nas maiores redes no Brasil. Organização \& Sociedade, [s. 1.], v. 15, n. 51, p. 665-686, 2009.

PIETSCH, Gotthard. Human capital measurement, ambiguity, and opportunism: actors between menace and opportunity. Zeitschrift fur Personalforschung, [s. 1.], v. 21, n. 3, p. 252-273, 2007.

ROCHA-PINTO, Sandra; DEL CARPIO, Gisele. Fatores críticos para a implantação do balanced scorecard: a visão de consultores organizacionais. BASE - Revista de Administração e Contabilidade da Unisinos, [s. 1.], v. 8, n. 4, p. 311-324, 2011.

RODRIGUES, Júlia Marçal; TORRES, Henrique Oswaldo da Gama. Eficiência/desempenho hospitalar e resultados da gestão de recursos humanos: uma aproximação possível? Gestão e Sociedade, [s. 1.], v. 9, n. 24, p. 1128-1142, 2015.

RUDIO, Franz Victor. Introdução ao projeto de pesquisa científica. Petrópolis: Vozes, 2014.

RUIZ, Paula Buck de Oliveira; PERROCA, Marcia Galan; JERICÓ, Marli de Carvalho. Custo da rotatividade da equipe de enfermagem em hospital de ensino. Revista da Escola de Enfermagem da USP, [s. 1.], v. 50, n. 1, p. 104-111, 2016.

SANCINETTI, Tânia Regina; SOARES, Alda Valéria Neves; LIMA, Antonio Fernandes Costa; SANTOS, Nanci Cristiano; MELLEIRO, Marta Maria; FUGULIN, Fernanda Maria Togeiro; GAIDZINSKI, Raquel Rapone. Taxa de absenteísmo da equipe de enfermagem como indicador de gestão de pessoas. Revista da Escola de Enfermagem da USP, [s. 1.], v. 45, n. 4, p. 1007-1012, 2011.

SILVA, Cláudio Cesar; LEITE, Nildes Raimunda Pitombo; RODRIGUES, Leonel Cezar. Gestão estratégica de pessoas e comprometimento organizacional em organizações hospitalares. Revista de Administração da UFSM, [s. 1.], v. 9, n. 2, p. 192-209, 2016.

SILVA, Dóris Marli Petry Paulo Da; MARZIALE, Maria Helena Palucci. Absenteísmo de trabalhadores de enfermagem em um hospital universitário. Revista Latino-Americana de Enfermagem, [s. 1.], v. 8, n. 5, p. 44-51, 2000. 
SILVEIRA, Victor Natanael Schwetter; FILHO, Antônio Del Maestro. Gestão estratégica de pessoas e desempenho organizacional: uma análise teórica. PRETEXTO, [s. 1.], v. 14, n. 1, p. 71-87, 2013.

SOARES, Thiago Coelho; MAZON, Gisele. Autoavaliação e seus drivers de mudança institucional baseado na capacidade absortiva. Revista Ibero-Americana de Estratégia, [s. 1.], v. 15, n. 4, p. 77-90, 2016.

STEEN, Adam; WELCH, Denice; MCCORMACK, Darcy. Conflicting conceptualizations of human resource accounting. Journal of Human Resource Costing \& Accounting, [s. 1.], v. 15, n. 4, p. 299-312, 2011.

TANURE, Betania; EVANS, Paul; CANÇADO, Vera L. As quatro faces de RH: analisando a performance da gestão de recursos humanos em empresas no Brasil. Revista de Administração Contemporânea, v. 14, n. 4, p. 594-614, 2010.

TINOCO, João Eduardo Prudêncio. Contribuição ao estudo da mensuração, avaliação e evidenciação de recursos humanos. Revista Contabilidade \& Finanças, v. 14, n. SPE, p. 66-78, 2003.

TINTI, Joel Adame; COSTA, Luciano Venelli.; VIEIRA, Almir Martins; CAPPELLOZZA, Alexandre. O Impacto das Políticas e Práticas de Recursos Humanos Sobre os Comportamentos de Cidadania Organizacional. Brazilian Business Review, v. 14, n. 6, p. 636-653, 2017.

ULRICH, Dave. Measuring Human Resources: an overview of practice. Human Resource Management, [s. 1.], v. 36, n. 3, p. 303-320, 1997.

ULRICH, Dave. Os campeões de recursos humanos. São Paulo: Futura, p. 84-98, 1998.

VAZQUEZ, Ana Claudia; OLTRAMARI, Andrea Poleto; TONON, Leonardo; SCHEFFER, Angela. Desempenho e carreira: produção acadêmica brasileira em administração no período de 2000 a 2010. Desenvolve Revista de Gestão do Unilasalle, [s. 1.], v. 2, n. 1, p. 17-38, 2013.

VIEIRA, Almir Martins. Cultura organizacional em instituições de ensino: mapeamento e análise descritivo-interpretativa da produção acadêmica (1990-2005). 2007. 237f. Tese (Doutorado em Educação) - Universidade Estadual Paulista (UNESP), Marília, 2007.

VIEIRA, Almir Martins.; FILENGA, Douglas. Gestão por competências: retórica organizacional ou prática da gestão de pessoas? Qualit@s (UEPB), v. 13, p. 1-12, 2012.

WARECH, Michael; TRACEY, J. Bruce. Evaluating the impact of human resources: identifying what matters. Cornell Hotel and Restaurant Administration Quarterly, [s. 1.], v. 45, n. 4, p. 376-387, 2004.

ZEHIR, Cemal; KOLE, Mahmut; GUROL, Yonca; KARABOGA, Tugba; KOLE, Mahmut. Strategic Human Resource Management and firm performance: the mediating role of entrepreneurial orientation. Procedia - Social and Behavioral Sciences, [s. 1.], v. 235, n. 1, p. 372-381, 2016.

Recebido em: 11-8-2019

Aprovado em: 22-11-2020

Avaliado pelo sistema double blind review.

Disponível em http://mjs.metodista.br/index.php/roc 